\title{
Uniform asymptotic approximation of the evanescent part of the Green's tensor
}

\author{
Henk F. Arnoldus*, John T. Foley \\ Department of Physics and Astronomy, Mississippi State University, P.O. Drawer 5167, Mississippi State, Mississippi 39762-5167, USA
}

Received 28 January 2002; accepted 13 March 2002

\begin{abstract}
The evanescent part of optical radiation from a localized source decays rapidly with distance. We have studied the asymptotic behavior of this radiation by means of the Green's tensor. This tensor can be expressed in terms of four auxiliary functions $M_{i}(q, \theta)$, with $q$ the (dimensionless) distance to the source and $\theta$ the polar angle. We have derived asymptotic approximations for these functions, with Bleistein's method, which hold uniformly for all angles $\theta$. Our result generalizes the result of Berry for the evanescent part of the free space scalar Green's function. We have illustrated graphically the accuracy of our approximation. (c) 2002 Elsevier Science B.V. All rights reserved.
\end{abstract}

PACS: $03.50 . \mathrm{De} ;$ 42.25.Bs

Keywords: Dipole radiation; Green's tensor; Evanescent waves; Asymptotic approximation

\section{Introduction}

The electromagnetic radiation field which is emitted by an oscillating electric dipole or an atom in free space is usually considered as an outgoing spherical wave, emanating from the location of the source [1]. In many practical applications, such as a radiating atom near a medium, this representation is not the most convenient due to boundary conditions at the planar interface. A solution to this problem is to represent the dipole field as a

\footnotetext{
${ }^{*}$ Corresponding author. Tel.: +1-662-325-2919; fax: +1-662325-8898.

E-mail addresses: arnoldus@ra.msstate.edu (H.F. Arnoldus), jtf1@ra.msstate.edu (J.T. Foley).
}

superposition of plane waves, each of which satisfies Maxwell's equations in free space. In this fashion, the plane waves can be matched across the boundary by means of the Fresnel coefficients, and this provides a representation of the solution of Maxwell's equations [2-6]. In such an approach, this superposition, referred to as the angular spectrum, consists of (radiative) traveling waves and of exponentially decaying (evanescent) waves. The traveling part of the electric field has been studied most extensively, since the far field, which is amenable to observation by macroscopic detectors, is predominantly determined by the traveling waves. This far field can be obtained from an angular spectrum representation by means of the method of stationary phase $[7,8]$, which yields an 
asymptotic approximation for the field at a large distance from the source.

The evanescent waves, which are generated by the source, decay in the $z$-direction and propagate in the $x y$-plane. Since these waves decay rapidly, they only seem to have significance for the optical near field in the vicinity of the source. When the source is located near an interface, the interaction between the source and the medium can be expected to depend strongly on the evanescent waves, and this has indeed been found experimentally [9-11]. On the other hand, it is known that the evanescent waves can survive in the far zone for observation points that are on the $z$-axis or in the $x y$-plane [12]. Since these directions seem to have zero angular measure, it was assumed that this phenomenon was merely a mathematical artifact. Recently, however, it was shown $[13,14]$ that there is a finite cylindrical region around the $z$ axis, with a diameter of about a fraction of a wavelength, where the evanescent waves extend into the far zone. Although the angular width of such a cylinder vanishes for large distances, its cross-section does not, and this might indicate that evanescent waves could be observable in the far field. More recently it was shown by Berry [15] that also in the neighborhood of the $x y$-plane the evanescent waves survive in the far zone over a finite cross-section. In this paper we generalize Berry's approach to derive a uniform asymptotic approximation for the evanescent part of the electric field of a dipole as a function of the distance $r$ to the source and uniform in the polar angle $\theta$.

\section{Green's tensor and angular spectrum}

The electric field of an electric dipole $\hat{\boldsymbol{\mu}}(\omega)$ at the origin of coordinates is most conveniently expressed in terms of the Green's tensor $\overleftrightarrow{\gamma}(\mathbf{r}, \omega)$ according to

$\hat{\mathbf{E}}(\mathbf{r}, \omega)=\frac{k_{0}^{3}}{4 \pi \varepsilon_{0}} \overleftrightarrow{\gamma}(\mathbf{r}, \omega) \cdot \hat{\boldsymbol{\mu}}(\omega)$.

The angular frequency $\omega$ indicates a Fourier time transform and the wave number $k_{0}$ equals $\omega / c$. Since all spatial dependence of the field is incorporated in the Green's tensor, which is indepen- dent of the source, it suffices to consider only this Green's tensor, defined by

$\overleftrightarrow{\gamma}(\mathbf{r}, \omega)=\left(\overleftrightarrow{\mathrm{I}}+\frac{1}{k_{0}^{2}} \nabla \nabla\right) \frac{\mathrm{e}^{\mathrm{i} k_{0} r}}{k_{0} r}$

We shall assume $r \neq 0$ throughout. For extended sources, the electric field can be found from (1) by superposition. When working out the derivatives in (2), terms which fall off as $r^{-3}, r^{-2}$ and $r^{-1}$ appear, and these represent the near, middle and far field, respectively. Each term is proportional to $\exp \left(\mathrm{i} k_{0} r\right)$ and therefore corresponds to an outgoing spherical wave.

The angular spectrum representation of $\overleftrightarrow{\gamma}(\mathbf{r}, \omega)$ can be obtained by means of Weyl's representation of the scalar Green's function [16]

$\frac{\mathrm{e}^{\mathrm{i} k_{0} r}}{r}=\frac{\mathrm{i}}{2 \pi} \int \mathrm{d}^{2} \mathbf{k}_{\|} \frac{\mathrm{e}^{\mathrm{i}\left(\mathbf{k}_{\|} \cdot \mathbf{r}+\beta|z|\right)}}{\beta}$.

Here, $\mathbf{k}_{\|}$is a vector in the $x y$-plane and the parameter $\beta$ is defined as $\beta=\left(k_{0}^{2}-k_{\|}^{2}\right)^{1 / 2}$ for $k_{\|}<k_{0}$ and $\beta=\mathrm{i}\left(k_{\|}^{2}-k_{0}^{2}\right)^{1 / 2}$ for $k_{\|}>k_{0}$. Expression (3) is a superposition of plane waves. When $k_{\|}<k_{0}$ the corresponding plane wave travels in the direction of the wave vector $\left(\mathbf{k}_{\|}, \beta\right)$ for $z>0$ and $\left(\mathbf{k}_{\|},-\beta\right)$ for $z<0$ without change of amplitude. When $k_{\|}>k_{0}$ the wave travels in the $x y$-plane in the direction of $\mathbf{k}_{\|}$, and decays exponentially in the $z$ direction. In this fashion, the angular spectrum integral (3) separates the scalar Green's function into a traveling and an evanescent part. When substituted into (2), the dyadic Green's function splits similarly. When we bring the derivatives under the integral and work out the resulting expression we find the compact representation

$\overleftrightarrow{\gamma}(\mathbf{r}, \omega)=\frac{\mathrm{i}}{2 \pi k_{0}} \int \mathrm{d}^{2} \mathbf{k}_{\|} \frac{1}{\beta}\left(\overleftrightarrow{\mathrm{I}}-\frac{1}{k_{0}^{2}} \mathbf{K K}\right) \mathrm{e}^{\mathrm{i} \mathbf{K} \cdot \mathbf{r}}$,

where we have set $\mathbf{K}=\mathbf{k}_{\|}+\beta \operatorname{sgn}(z) \mathbf{e}_{z}$ for the wave vector of the partial waves.

\section{Evanescent part}

The evanescent part of the Green's tensor now follows from (4) by restricting the integration over the $\mathbf{k}_{\|}$-plane to the region outside the circle $k_{\|}=k_{0}$. When using polar coordinates, the angular 
integration can be performed easily, leading to Bessel functions $J_{n}(x)$. For the radial integral we make the change of variables $k_{\|}=k_{0}\left(1+u^{2}\right)^{1 / 2}$, and we shall adopt dimensionless coordinates with $1 / k_{0}$ as length scale. The distance to the origin (in configuration space) will be indicated by $q=k_{0} r$, and the $z$-coordinate becomes $\zeta=k_{0} z$. It is also convenient to introduce $\rho$ as the dimensionless polar coordinate in the $x y$-plane (distance to the $z$-axis), e.g., $\rho=k_{0}\left(x^{2}+y^{2}\right)^{1 / 2}$. Putting everything together then yields the following expression for the evanescent part of the Green's tensor [17]:

$$
\begin{aligned}
\overleftrightarrow{\gamma}(\mathbf{r}, \omega)^{\mathrm{ev}}= & \frac{1}{2}\left(\stackrel{\leftrightarrow}{\mathrm{I}}+\mathbf{e}_{z} \mathbf{e}_{z}\right) M_{a}(q, \theta) \\
& +\frac{1}{2}\left(\stackrel{\leftrightarrow}{\mathrm{I}}-\mathbf{e}_{z} \mathbf{e}_{z}-2 \hat{\mathbf{r}}_{\|} \hat{\mathbf{r}}_{\|}\right) M_{b}(q, \theta) \\
& +\frac{1}{2} \operatorname{sgn}(\zeta)\left(\hat{\mathbf{r}}_{\|} \mathbf{e}_{z}+\mathbf{e}_{z} \hat{\mathbf{r}}_{\|}\right) M_{c}(q, \theta) \\
& +\frac{1}{2}\left(\stackrel{\leftrightarrow}{\mathrm{I}}-3 \mathbf{e}_{z} \mathbf{e}_{z}\right) M_{d}(q, \theta) .
\end{aligned}
$$

Here $\hat{\mathbf{r}}_{\|}$is the radial unit vector in the $x y$-plane and $\operatorname{sgn}(\zeta)$ indicates the sign of $\zeta$. The coordinate dependence of this tensor appears entirely through a set of four auxiliary functions, which are all of the form

$$
M(q, \theta)=\int_{0}^{\infty} \mathrm{d} u f(u) J_{n}\left(\rho \sqrt{1+u^{2}}\right) \mathrm{e}^{-u|\zeta|} .
$$

They differ in their $f(u)$ and the order $n$ of the Bessel function, as shown in Table 1. The $q$ and $\theta$ dependence enters here through $\rho=q \sin \theta$ and $\zeta=q \cos \theta$. These four integrals can be expressed in closed form by means of Lommel functions [18], but the resulting expressions are cumbersome $[17,19,20]$ and not very suitable for the study of the behavior of the tensor for large $q$.

\section{Asymptotic approximation}

In this section we derive an asymptotic approximation for integrals of the general type (6), for $q$ large, $\theta$ arbitrary. The $q$-dependence appears in the exponential through $\zeta=q \cos \theta$ and in the argument of the Bessel function as $\rho=q \sin \theta$. For $q$ large we then also have $\rho$ large, provided we exclude the $z$-axis, $\theta=0$ or $\pi$. As the first step we use the asymptotic approximation of the Bessel functions for large argument:

$J_{n}(x) \approx \sqrt{\frac{2}{\pi x}} \cos \left[x-\frac{\pi}{4}(2 n+1)\right]$.

Then (6) can be approximated accordingly:

$M(q, \theta) \approx \sqrt{\frac{2}{\pi \rho}} \operatorname{Re}\left[\mathrm{e}^{-(\mathrm{i} \pi / 4)(2 n+1)} m(q, \theta)\right]$,

where we have set

$m(q, \theta)=\int_{0}^{\infty} \mathrm{d} u \frac{f(u)}{\left(1+u^{2}\right)^{1 / 4}} \mathrm{e}^{q w(u)}$,

with

$w(u)=-u|\cos \theta|+i \sin \theta \sqrt{1+u^{2}}$.

We therefore need an asymptotic approximation for integrals of the type (9). In this form, the large parameter $q$ only appears in the exponent and only as an overall factor, which is a great simplification.

The critical points of the integral (9) are the lower limit $u=0$, and a possible saddle point of the function $w(u)$ in the complex $u$-plane. For a saddle point we have $w^{\prime}(u)=0$, and it is easily verified from (10) that this saddle point is located at $u_{0}=-\mathrm{i}|\cos \theta|$ for a given $\theta$. Although the integration contour does not pass through the saddle point, for $\theta \rightarrow \pi / 2$ the saddle point approaches the

Table 1

Function $f(u)$, order $n$ and parameters for the four integrals

\begin{tabular}{lllll}
\hline & $M_{a}(q, \theta)$ & $M_{b}(q, \theta)$ & $M_{c}(q, \theta)$ & $M_{d}(q, \theta)$ \\
\hline$f(u)$ & 1 & $-\left(1+u^{2}\right)$ & $2 u \sqrt{1+u^{2}}$ & $-u^{2}$ \\
$n$ & 0 & 2 & 1 & 0 \\
$f(0)$ & 1 & -1 & 0 & 0 \\
$f\left(u_{0}\right)$ & 1 & $-\sin ^{2} \theta$ & $-\mathrm{i}|\sin 2 \theta|$ & $\cos ^{2} \theta$ \\
$f^{\prime}(0)$ & 0 & 0 & 2 & 0 \\
\hline
\end{tabular}


integration endpoint $u=0$ from the negative imaginary axis. It can then be anticipated that this saddle point gives a contribution which can be comparable to the contribution from the endpoint. For situations like this, an asymptotic approximation can be obtained with Bleistein's method [2123], and this was applied recently by Berry [15] to find a uniform asymptotic approximation in $\theta$ for the scalar Green's function (our $M_{a}(q, \theta)$ ), which smoothly varies in the neighborhood of $\theta=\pi / 2$.

In Bleistein's method, a change of variables $u \rightarrow t$ is made, and in such a way that the exponent $w(u)$ is mapped onto a quadratic form. The appropriate transformation here is

$w(u)=-\frac{1}{2} t^{2}-\alpha t+\mathrm{i} \sin \theta$,

with

$\alpha=(1+i) \sqrt{1-\sin \theta}$.

In this way, the new saddle point appears at $t=-\alpha$. The contour of integration $C$ in the complex $t$-plane starts at the origin, lies in the fourth quadrant and approaches a line under at most $45^{\circ}$ with the positive real axis, as shown in Fig. 1. Next we make a linear approximation of the integrand, excluding the exponential factor. We set

$\frac{f(u)}{\left(1+u^{2}\right)^{1 / 4}} \frac{\mathrm{d} u}{\mathrm{~d} t} \approx a_{0}+a_{1} t$

with $u=u(t)$, and the constants $a_{0}$ and $a_{1}$ are chosen such that this approximation is exact in the

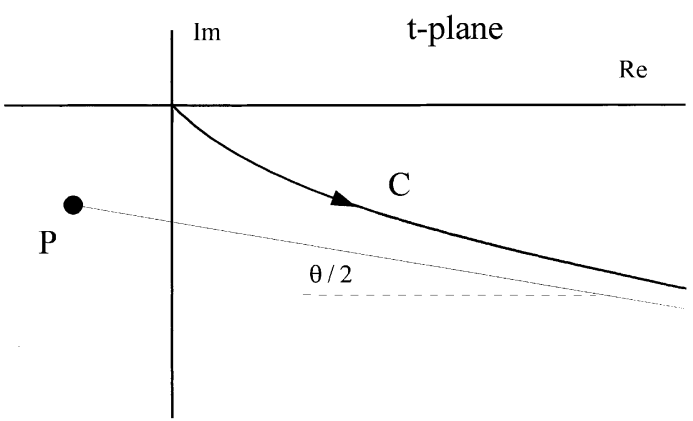

Fig. 1. Illustration of the contour of integration $C$ for the integral (18) in the complex $t$-plane. The curve approaches the straight line through the saddle point $P$, and this line slopes down under an angle of $\theta / 2$ (for $\theta<\pi / 2$; otherwise the angle is $(\pi-\theta) / 2)$. The parametrization of $C$ follows from solving transformation (11) for $t(u)$. critical points $t=0$ and $t=-\alpha$. From the transformation (11) we see that

$\frac{\mathrm{d} u}{\mathrm{~d} t}=-\frac{t+\alpha}{w^{\prime}(u)}$,

and since $t=0$ corresponds to $u=0$ we find from (13) with $t=0$ :

$a_{0}=\frac{(1+\mathrm{i}) f(0)}{\sqrt{1+\sin \theta}}$.

For $t=-\alpha$, the right-hand side of (14) has to be evaluated with a limit procedure. This gives $(\mathrm{d} z / \mathrm{d} t)_{t=-\alpha}=\sqrt{\mathrm{i}} \sin \theta$, and then with (13) we find

$a_{1}=\frac{f(0)-f\left(u_{0}\right) b(\theta)}{|\cos \theta|}$,

where we have set

$b(\theta)=\sqrt{\frac{1}{2} \sin \theta(1+\sin \theta)}$.

The values of $f(0)$ and $f\left(u_{0}\right)$ for the various integrals are listed in Table 1.

With the approximation (13) the integral (9) becomes in the $t$-plane

$m(q, \theta) \approx \mathrm{e}^{\mathrm{i} \rho} \int_{C} \mathrm{~d} t\left(a_{0}+a_{1} t\right) \mathrm{e}^{-(1 / 2) t^{2}-\alpha t}$.

The integrand is analytic and with Cauchy's theorem the contour can be brought back to the real axis. The remaining integral can then be expressed in terms of the complementary error function. The asymptotic approximation for $m(q, \theta)$ is then found to be, after some rearrangements,

$$
\begin{aligned}
& m(q, \theta) \approx \frac{\mathrm{e}^{\mathrm{i} \rho}}{|\zeta|}\left[f(0)-f\left(u_{0}\right) b(\theta)\right] \\
& \quad+f\left(u_{0}\right) \sqrt{\frac{\pi \sin \theta}{2 q}} \mathrm{e}^{\mathrm{i}(q+(\pi / 4))} \operatorname{erfc}[\sqrt{\mathrm{i}(q-\rho)}],
\end{aligned}
$$

and with (8) we find for $M(q, \theta)$

$$
\begin{aligned}
M(q, \theta) \approx & \frac{1}{|\zeta|} \sqrt{\frac{2}{\pi \rho}} \\
& \times \operatorname{Re}\left\{\left[f(0)-f\left(u_{0}\right) b(\theta)\right] \mathrm{e}^{\mathrm{i}(\rho-(\pi / 4)(2 n+1))}\right\} \\
& +\frac{1}{q} \operatorname{Re}\left\{f\left(u_{0}\right) \mathrm{e}^{\mathrm{i}(q-(n \pi / 2))} \operatorname{erfc}[\sqrt{\mathrm{i}(q-\rho)}]\right\} .
\end{aligned}
$$


With the values of $f(0)$ and $f\left(u_{0}\right)$ from Table 1 , this determines the asymptotic approximation of the four auxiliary functions in the Green's tensor, uniform through $\theta=\pi / 2$.

Expression (20) is constructed to be uniform near $\theta=\pi / 2$. This implies that it should hold for all $\theta$, except, perhaps, for $\theta$ approaching 0 or $\pi$. First we observe that for $\theta \rightarrow \pi / 2$ we have $|\zeta| \rightarrow 0$, and it appears that the first term on the right-hand side is undefined. However, when we approach the $x y$-plane we have $u_{0} \rightarrow 0$, and therefore $\left[f(0)-f\left(u_{0}\right) b(\theta)\right] \rightarrow 0$. Apparently, the value near the $x y$-plane has to be investigated with a limit procedure. After we expand $f\left(u_{0}\right)$ in a Taylor series around $u=0$, we find the following limit:

$\lim _{\theta \rightarrow \pi / 2} \frac{1}{|\cos \theta|}\left[f(0)-f\left(u_{0}\right) b(\theta)\right]=f^{\prime}(0)$ i.

Furthermore, in this limit we have $\rho=q$, and $\operatorname{erfc}(0)=1$, and we can use that $f(0)$ and $f^{\prime}(0)$ are real. The result (20) then reduces to

$$
\begin{aligned}
M(q, \pi / 2) \approx & \frac{f(0)}{q} \cos \left(q-\frac{n \pi}{2}\right)+\frac{1}{q} \sqrt{\frac{2}{\pi q}} f^{\prime} \\
& \times(0) \cos \left[q-\frac{\pi}{4}(2 n-1)\right] .
\end{aligned}
$$

This shows that in the $x y$-plane the leading term will in general be $\mathcal{O}\left(q^{-1}\right)$, which is a far field contribution. Only if $f(0)=0$, the leading term becomes $\mathcal{O}\left(q^{-3 / 2}\right)$, which falls off faster than the far field.

\section{Uniform asymptotic approximation}

For $\theta \neq \pi / 2$ and $q$ large, we have $\operatorname{erfc}[\sqrt{\mathrm{i}(q-\rho)}]=\operatorname{erfc}[\sqrt{\mathrm{i} q(1-\sin \theta)}] \rightarrow 0, \quad$ and only the first term on the right-hand side of (20) survives. This term is $\mathcal{O}\left(q^{-3 / 2}\right)$, provided not both $f(0)$ and $f\left(u_{0}\right)$ vanish. If $\theta$ approaches 0 or $\pi$, approximation (20) becomes invalid, since in this limit we have $\rho \rightarrow 0$ whereas we used explicitly that $\rho$ is large. On the other hand, for $\rho$ large, both sides of (7), with $x=\rho$, are asymptotically equivalent. On the right-hand side of (20), the term which is proportional to $f(0)$ contains exactly the approximation of $J_{n}(\rho)$, so as long as $\rho$ is large we can replace this part again with $J_{n}(\rho)$. The result then becomes

$$
\begin{aligned}
M(q, \theta) \approx & \frac{f(0)}{|\zeta|} J_{n}(\rho)-\frac{1}{q^{3 / 2}} \frac{1}{\sqrt{\pi(1-\sin \theta)}} \\
& \operatorname{Re}\left\{f\left(u_{0}\right) \mathrm{e}^{\mathrm{i}\left[\rho-\frac{\pi}{4}(2 n+1)\right]}\right\} \\
& +\frac{1}{q} \operatorname{Re}\left\{f\left(u_{0}\right) \mathrm{e}^{\mathrm{i}\left(q-\frac{n \pi}{2}\right)} \operatorname{erfc}[\sqrt{\mathrm{i}(q-\rho)}]\right\},
\end{aligned}
$$

where we have used (17). With some further rearrangements this can be written as

$$
\begin{aligned}
M(q, \theta) \approx & \frac{f(0)}{|\zeta|} J_{n}(\rho)+\frac{1}{q} \operatorname{Re}\left(f\left(u_{0}\right) \mathrm{e}^{\mathrm{i}\left(q-\frac{n \pi}{2}\right)}\right. \\
& \times\{\operatorname{erfc}[\sqrt{\mathrm{i}(q-\rho)}] \\
& \left.\left.-\frac{1}{\sqrt{\pi \mathrm{i}(q-\rho)}} \mathrm{e}^{-\mathrm{i}(q-\rho)}\right\}\right)
\end{aligned}
$$

We now claim that in this form the asymptotic approximation is uniform for all $\theta$. To show this, we go back to (6) and integrate by parts. This yields

$$
\begin{aligned}
M(q, \theta)= & \frac{f(0)}{|\zeta|} J_{n}(\rho)+\frac{1}{|\zeta|} \int_{0}^{\infty} \mathrm{d} u \mathrm{e}^{-u|\zeta|} \\
& \times \frac{\mathrm{d}}{\mathrm{d} u}\left[f(u) J_{n}\left(\rho \sqrt{1+u^{2}}\right)\right]
\end{aligned}
$$

as an exact integral. Repeated integration by parts then gives an asymptotic series in $1 /|\zeta|$, which holds for $|\zeta|$ large, but $\rho$ arbitrary. We see that the first term on the right-hand side of (24) is the first term in this asymptotic series. On the other hand, with the asymptotic series for the complementary error function [24] we have

$\operatorname{erfc}(z)-\frac{1}{z \sqrt{\pi}} \mathrm{e}^{-z^{2}}=-\frac{1}{2 z^{3} \sqrt{\pi}} \mathrm{e}^{-z^{2}}\left[1+\mathcal{O}\left(\frac{1}{z^{2}}\right)\right]$,

needed for $z=\sqrt{\mathrm{i}(q-\rho)}$. For $q$ large, this becomes $\mathcal{O}\left(q^{-3 / 2}\right)$, and the additional $1 / q$ makes the second term on the right-hand side of (24) order $q^{-5 / 2}$. Since the first term is $\mathcal{O}\left(q^{-3 / 2}\right)$, the second term can be neglected for $|\zeta|$ large. This shows that (24) and (25) are asymptotically equivalent for $|\zeta|$ 
large. Since (24) was already shown to be correct for $\rho$ large, this proves that it holds for $q$ large, any $\theta$, making this a true uniform asymptotic approximation. It should be noted though that the case $\theta \rightarrow \pi / 2$ still has to be considered as a limit, with the result given by (22), or equivalently by

$M(q, \pi / 2) \approx \frac{f(0)}{q} \cos \left(q-\frac{n \pi}{2}\right)-\frac{f^{\prime}(0)}{q} J_{n+1}(q)$.

\section{The $M(q, \theta)$ functions of the Green's tensor}

The general result (24) can be simplified further for the four integrals $M_{a}, M_{b}, M_{c}$ and $M_{d}$ in $\overleftrightarrow{\gamma}(\mathbf{r}, \omega)^{\mathrm{ev}}$, Eq. (5). To this end, we introduce the universal function of $q$ and $\theta$ :

$$
\begin{aligned}
n(q, \theta)=|\cos \theta| \operatorname{Re}( & \mathrm{e}^{\mathrm{i} q}\left\{\frac{1}{\sqrt{\pi \mathrm{i}(q-\rho)}} \mathrm{e}^{-\mathrm{i}(q-\rho)}\right. \\
& -\operatorname{erfc}[\sqrt{\mathrm{i}(q-\rho)}]\}) .
\end{aligned}
$$

The overall factor of $|\cos \theta|$ is included to keep this function finite for $\theta \rightarrow \pi / 2$. We can rewrite (28) as

$$
\begin{aligned}
n(q, \theta)= & \sqrt{\frac{1+\sin \theta}{\pi q}} \cos \left(\rho-\frac{\pi}{4}\right) \\
& -|\cos \theta| \operatorname{Re}\left\{\mathrm{e}^{\mathrm{i} q} \operatorname{erfc}[\sqrt{\mathrm{i}(q-\rho)}]\right\},
\end{aligned}
$$

from which we find for $\theta \rightarrow \pi / 2$ :

$n(q, \pi / 2)=\sqrt{\frac{2}{\pi q}} \cos \left(q-\frac{\pi}{4}\right)$,

which is asymptotically equal to $J_{0}(q)$. It then follows immediately that near $\theta=\pi / 2$ this function is of order $q^{-1 / 2}$. On the other hand, from (28) and (26) we see that $n(q, \theta)=\mathcal{O}\left(q^{-3 / 2}\right)$ for $\theta \neq \pi / 2$. Function $n(q, \theta)$ is shown in Fig. 2 as a function of $\theta$, for various values of $q$.

With (24) and the parameters in Table 1 we now find the asymptotic result for the parts of the Green's tensor

$M_{a}(q, \theta) \approx \frac{1}{|\zeta|}\left[J_{0}(\rho)-n(q, \theta)\right]$

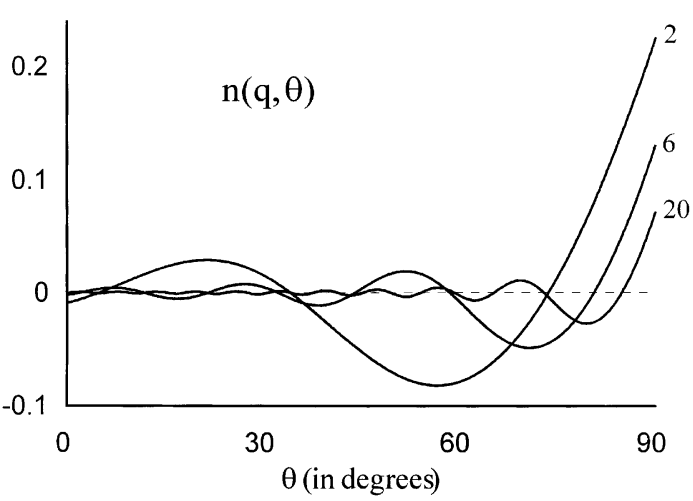

Fig. 2. Graphs of the universal function $n(q, \theta)$, defined by (28), for $q=2,6$ and 20 .

$M_{b}(q, \theta) \approx-\frac{1}{|\zeta|}\left[J_{2}(\rho)+\sin ^{2} \theta n(q, \theta)\right]$,

$M_{c}(q, \theta) \approx \frac{2 \sin \theta}{q} n(q, \theta)$,

$M_{d}(q, \theta) \approx-\frac{|\cos \theta|}{q} n(q, \theta)$,

with $\rho=q \sin \theta$ as the arguments of the Bessel functions. For $\theta=\pi / 2$ we obtain from (27) the limiting values

$$
\begin{aligned}
& M_{a}(q, \pi / 2) \approx \frac{\cos q}{q}, \\
& M_{b}(q, \pi / 2) \approx \frac{\cos q}{q}, \\
& M_{c}(q, \pi / 2) \approx-\frac{2}{q} J_{2}(q), \\
& M_{d}(q, \pi / 2) \approx 0 .
\end{aligned}
$$

This shows that near the $x y$-plane $M_{a}$ and $M_{b}$ are $\mathcal{O}\left(q^{-1}\right)$, e.g., they contribute to the far field, whereas $M_{c}$ decays as $\mathcal{O}\left(q^{-3 / 2}\right)$ and $M_{d}$ decays even faster. It is interesting to note that in the $x y$-plane the exact values are known for all $q[13,17]$. It appears that the expressions above for $M_{a}$ and $M_{c}$ are exact for all $q$, and the other two have an unresolved part of $\mathcal{O}\left(q^{-2}\right)$.

For $\theta \neq \pi / 2$, we have $n(q, \theta)=\mathcal{O}\left(q^{-3 / 2}\right)$, and each term in (31)-(34) containing $n(q, \theta)$ is $\mathcal{O}\left(q^{-5 / 2}\right)$. Since the uniform asymptotic approximation is only accurate up to $\mathcal{O}\left(q^{-3 / 2}\right)$, these terms should be neglected. We then find 
$M_{a}(q, \theta) \approx \frac{1}{|\zeta|} J_{0}(\rho)$

$M_{b}(q, \theta) \approx-\frac{1}{|\zeta|} J_{2}(\rho)$,

$M_{c}(q, \theta) \approx M_{d}(q, \theta) \approx 0$.

Since the Bessel functions are $\mathcal{O}\left(q^{-1 / 2}\right)$ for $\rho$ large, we see that $M_{a}$ and $M_{b}$ are $\mathcal{O}\left(q^{-3 / 2}\right)$ whereas the other two functions vanish. This typical $\mathcal{O}\left(q^{-3 / 2}\right)$ dependence has been found before [14]. For large $\rho$ we also have $J_{2}(\rho) \approx-J_{0}(\rho)$, as follows from (7), and therefore we have $M_{b}(q, \theta) \approx M_{a}(q, \theta)$. From the point of view of the Green's tensor, near the $x y$-plane it has a far field part, $\mathcal{O}\left(q^{-1}\right)$, and this goes over smoothly into the $\mathcal{O}\left(q^{-3 / 2}\right)$ dependence away from the $x y$-plane. Apparently, there is a layer near the $x y$-plane where the evanescent waves survive in the far field. This region is determined by the function $n(q, \theta)$. This function is $\mathcal{O}\left(q^{-1 / 2}\right)$ whenever the $\operatorname{erfc}(z)$ has not yet reached its asymptotic value given by (26), and this is for $q-\rho \lesssim 1$. Since $q=\left(\rho^{2}+\zeta^{2}\right)^{1 / 2}$, the thickness of the layer in the $z$-direction is about $|\zeta| \approx \sqrt{\rho}$, for a given $\rho$. It is interesting to notice that the layer thickness becomes larger for greater distances from the source. The angular width $\Delta \theta$, however, is $\Delta \theta \approx|\zeta| / \rho$ and this is $\Delta \theta \approx 1 / \sqrt{\rho}$. Consequently, the angular width vanishes for $\rho$ large, even though the corresponding layer thickness grows indefinitely with $\sqrt{\rho}$.

The typical $\mathcal{O}\left(q^{-3 / 2}\right)$ behavior from the previous paragraph is based on the assumption of $\rho$ large, which is equivalent to $\theta \neq 0$ or $\pi$. When $\theta \rightarrow 0$ or $\pi$, e.g., when the field point becomes close to the $z$ axis, the character of the Bessel functions changes. We then have $J_{0}(\rho) \approx 1$ and $J_{2}(\rho) \approx 0$. Then (39) becomes

$M_{a}(q, 0$ or $\pi) \approx \frac{1}{q}$,

and the other three vanish entirely. Also for the $z$ axis, the $M(q, \theta)$ integrals can be evaluated in closed form, and it appears that (42) holds exactly. It can also be shown that $M_{a}=M_{b}=0$, so this asymptotic result is also exact. Only the exact value for $M_{d}$ has an $\mathcal{O}\left(q^{-3}\right)$ contribution on the $z$ axis, which is not resolved properly with the asymptotic approximation. We notice that also on the $z$-axis the leading contribution is of the far field type, whereas a distance away from the $z$-axis the $q$ dependence is $\mathcal{O}\left(q^{-3 / 2}\right)$. The transition here is determined by the value of $\rho$ where the asymptotic behavior of $J_{0}(\rho)$ and $J_{2}(\rho)$ sets in. This occurs at $\rho \approx 1$, so at a radial distance of about a wavelength from the $z$-axis. Therefore, the far-field behavior of the evanescent waves is dominant in a cylindrical region around the $z$-axis. Again, the corresponding angular width vanishes asymptotically, but the cross-section of the cylinder remains finite for all $|\zeta|$, and the radius of this cylinder is of the order of about a wavelength.

Fig. 3 shows the exact $M_{a}(q, \theta)$ and its asymptotic approximation (31) as a function of $\theta$ for $q=2 \pi$. Here the distance to the source is only one wavelength, and the approximation already seems excellent, except close to $\pi / 2$. The limiting value (35) for $\theta \rightarrow \pi / 2$ is actually exact for all $q$, and therefore one would expect that the agreement near $\pi / 2$ would be better. However, (35) follows from (31) under the assumption that $q$ is large, and apparently $q=2 \pi$ is too small for (35) to set in. Fig. 4 shows that the approximation near $\pi / 2$ improves rapidly with increasing $q$. A result for $M_{b}(q, \theta)$ is shown in Fig. 5, and it appears that the approximation near $\theta=\pi / 2$ is less accurate than the corresponding approximation for $M_{a}(q, \theta)$ from Fig. 4 (both are shown for $q=10 \pi$ ). As the

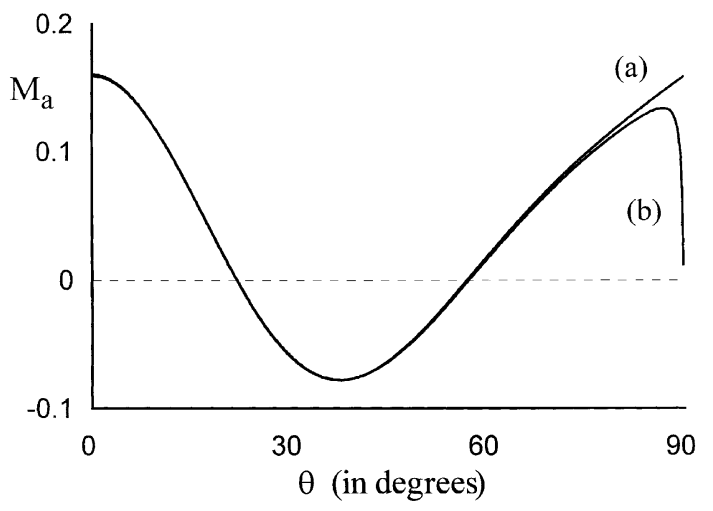

Fig. 3. Curve (a) shows the exact $M_{a}(q, \theta)$ for $q=2 \pi$ and curve (b) is the asymptotic approximation from (31). The deviation near $\theta=\pi / 2$ is a result of the fact that $q$ is not yet large enough for the asymptotic approximation to be accurate for this angle. 


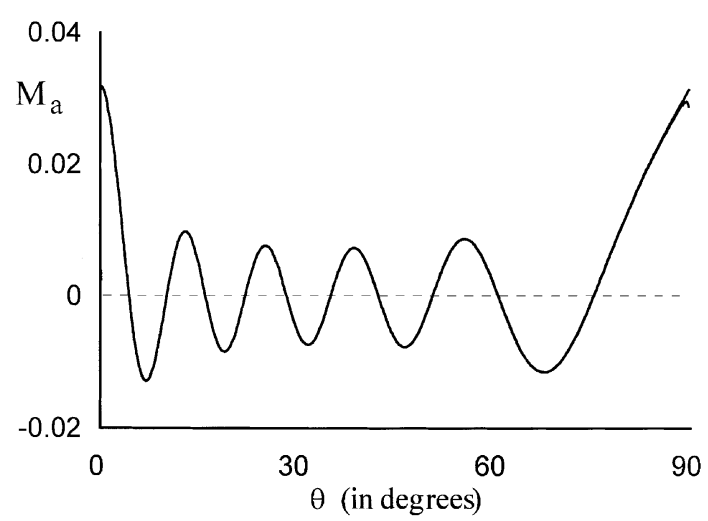

Fig. 4. The exact $M_{a}(q, \theta)$ for $q=10 \pi$ and its approximation. As compared to Fig. 3, the approximation is now also virtually perfect near $\theta=\pi / 2$.

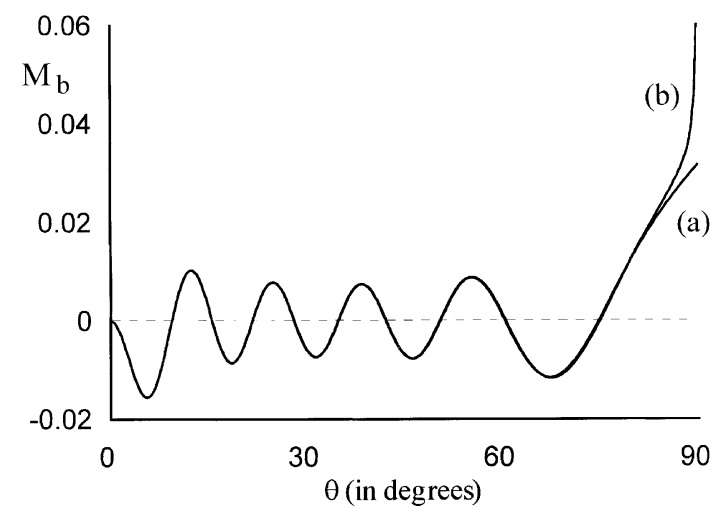

Fig. 5. Curves (a) and (b) illustrate the exact and approximate $M_{b}(q, \theta)$, respectively, for $q=10 \pi$.

value of $q$ increases, the approximation for $M_{b}(q, \theta)$ improves correspondingly. The approximation for $M_{c}(q, \theta)$ is $\mathcal{O}\left(q^{-3 / 2}\right)$ near the $x y$-plane but $\mathcal{O}\left(q^{-5 / 2}\right)$ away from the $x y$-plane, as follows from (33). Since the asymptotic approximation is constructed to be accurate up to order $q^{-3 / 2}$, but not further, the approximation for $M_{c}(q, \theta)$ can only be expected to be accurate near $\pi / 2$, but not for smaller angles. Fig. 6 shows that this is indeed the case. It is interesting to notice that here we do not see the slight deviation near $\pi / 2$ due to the finite value of $q$, as in Figs. 3 and 4 . The function $M_{d}(q, \theta)$ is $\mathcal{O}\left(q^{-5 / 2}\right)$ for $\theta \neq \pi / 2$, which is asymptotically negligible. Near the $x y$-plane it is $\mathcal{O}\left(q^{-3 / 2}\right)$, as seen from (34), but the overall $\cos \theta$

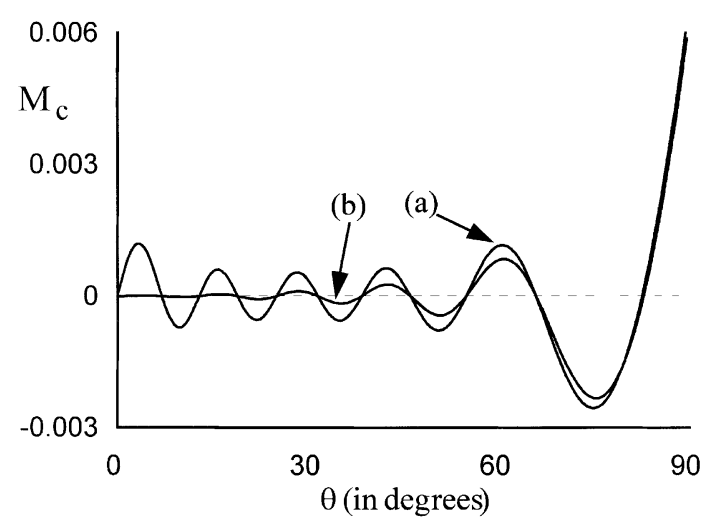

Fig. 6. Exact and approximate function $M_{c}(q, \theta)$ for $q=10 \pi$. This function only approximates the exact result in the neighborhood of $\theta=\pi / 2$.

makes $M_{d}(q, \theta)$ vanish for $\theta=\pi / 2$. Therefore, $M_{d}(q, \theta)$ can be neglected for all $\theta$.

The evanescent part of the Green's tensor follows after substitution of $M_{a}, M_{b}$ and $M_{c}$ from (31)-(33) into expression (5) for $\overleftrightarrow{\gamma}(\mathbf{r}, \omega)^{\text {ev }}$. To leading order, the tensor becomes $\mathcal{O}\left(q^{-1}\right)$ near the $z$-axis and near the $x y$-plane, and $\mathcal{O}\left(q^{-3 / 2}\right)$ elsewhere. These leading order-contributions come from $M_{a}$ and $M_{b}$ only, and it might appear that $M_{c}$, which is $\mathcal{O}\left(q^{-3 / 2}\right)$ near the $x y$-plane and negligible elsewhere, can be neglected entirely. This is however not the case. The tensor $\overleftrightarrow{\gamma}(\mathbf{r}, \omega)^{\text {ev }}$ couples the Cartesian coordinates of the dipole moment to the Cartesian coordinates of the electric field, as shown in (1). As seen in (5), the $M$ functions couple different components of the two vectors. For instance, let $\hat{\boldsymbol{\mu}}(\omega) \propto \mathbf{e}_{x}$, consider a field point on the $x$-axis, $\mathbf{r}=x \mathbf{e}_{x}$, and then evaluate the $z$ component of the electric field. This gives a term of the form

$$
\begin{aligned}
\mathbf{e}_{z} \cdot\left[\overleftrightarrow{\gamma}\left(x \mathbf{e}_{x}, \omega\right)^{\mathrm{ev}} \cdot \mathbf{e}_{x}\right] & =\frac{1}{2} \operatorname{sgn}(\zeta) M_{c}(q, \pi / 2) \\
& =-\frac{\operatorname{sgn}(\zeta)}{q} J_{2}(q),
\end{aligned}
$$

and this only contains the function $M_{c}$. Since this $\mathcal{O}\left(q^{-3 / 2}\right)$ term is the leading term for this field component, it should be retained, even though the order of the entire tensor at this field point is $\mathcal{O}\left(q^{-1}\right)$. 


\section{Conclusions}

Electromagnetic radiation emitted by a localized source is a superposition of traveling and evanescent waves, when represented by an angular spectrum. We have studied the asymptotic behavior, for large distances from the source, of the evanescent component by means of the Green's tensor. An asymptotic approximation was derived, which holds uniformly for all observation (or emission) angles. Our result generalizes earlier expressions for the scalar Green's function, which only held for large $z$-coordinates or only far away from the $z$-axis. The asymptotic approximations of the four auxiliary functions $M_{a}(q, \theta), M_{b}(q, \theta)$, $M_{c}(q, \theta)$ and $M_{d}(q, \theta)$ that occur in the Green's tensor could all be expressed in terms of a single, universal function $n(q, \theta)$ and low-order Bessel functions, as shown in (31)-(34). By considering the $q$-dependence of these functions, we have identified the contribution of each to the far field. It appeared that only $M_{a}$ and $M_{b}$ contribute to the far, $\mathcal{O}\left(q^{-1}\right)$, field, and only near the $z$-axis and near the $x y$-plane. Functions $M_{a}(q, \theta), M_{b}(q, \theta)$ and $M_{c}(q, \theta)$ contribute all to the more typical $\mathcal{O}\left(q^{-3 / 2}\right)$ behavior of evanescent radiation. It appeared that function $M_{d}(q, \theta)$ is asymptotically negligible for all angles. We have illustrated numerically that already for a few wavelengths away from the source our asymptotic approximation is accurate within graphing resolution.

\section{References}

[1] J.D. Jackson, in: Classical Electrodynamics, second ed., Wiley, New York, 1975, p. 395.
[2] W. Lukosz, R.E. Kunz, J. Opt. Soc. Am. 67 (1977) 16071615.

[3] J.E. Sipe, Surf. Sci. 105 (1981) 489-504.

[4] J.M. Wiley, J.E. Sipe, Phys. Rev. A 30 (1984) 1185-1193.

[5] J.E. Sipe, H.M. van Driel, J.F. Young, Can. J. Phys. 63 (1985) 104-113.

[6] H.F. Arnoldus, T.F. George, Phys. Rev. A 43 (1991) 3675-3689.

[7] M. Born, E. Wolf, in: Principles of Optics, seventh (expanded) ed., Cambridge University Press, Cambridge, 1999, p. 890.

[8] G.C. Sherman, J.J. Stamnes, É. Lalor, J. Math. Phys. 17 (1976) 760-776.

[9] R.R. Chance, A. Prock, R. Silbey, J. Chem. Phys. 60 (1974) 2744-2748.

[10] R.R. Chance, A.H. Miller, A. Prock, R. Silbey, J. Chem. Phys. 63 (1975) 1589-1595.

[11] R.R. Chance, A. Prock, R. Silbey, Adv. Chem. Phys. 39 (1978) 1-65.

[12] E. Wolf, J.T. Foley, Opt. Lett. 23 (1998) 16-18.

[13] T. Setälä, M. Kaivola, A.T. Friberg, Phys. Rev. E 59 (1999) 1200-1206.

[14] A.V. Shchegrov, P.S. Carney, J. Opt. Soc. Am. A 16 (1999) 2583-2584.

[15] M.V. Berry, J. Mod. Opt. 48 (2001) 1535-1541.

[16] L. Mandel, E. Wolf, Optical Coherence and Quantum Optics, Cambridge University Press, Cambridge, 1995, Section 3.2.4.

[17] H.F. Arnoldus, J.T. Foley, J. Opt. Soc. Am. A, 2002, in press.

[18] G.N. Watson, in: A Treatise on the Theory of Bessel Functions, Cambridge University Press, Cambridge, 1922, p. 537.

[19] D.C. Bertilone, J. Mod. Opt. 38 (1991) 865-875.

[20] D.C. Bertilone, J. Mod. Opt. 38 (1991) 1531-1536.

[21] F.W.J. Olver, in: Asymptotics and Special Functions, Academic Press, New York, 1974, p. 344.

[22] N. Bleistein, R.A. Handelsman, in: Asymptotic Expansions of Integrals, Dover, New York, 1986, p. 380.

[23] R. Wong, in: Asymptotic Approximations of Integrals, Academic Press, Boston, 1989, p. 410.

[24] M. Abramowitz, I.A. Stegun, in: Handbook of Mathematical Functions, Dover, New York, 1970, p. 298. 